\title{
Metastatic Squamous Cell Carcinoma of the Cervix Secreting Ectopic Serum Beta Human Chorionic Gonadotropin
}

\author{
Michelle E-Jyn Kwek ${ }^{\mathrm{a}, \mathrm{c}}$, Yu Hui Limª, Sheow Lei Limº, \\ Wai Loong Wong ${ }^{\mathrm{b}}$
}

\begin{abstract}
An elevated serum beta human chorionic gonadotropin ( $\beta$-hCG) level in a female patient usually demonstrates the presence of a pregnancy or to a lesser extent, gestational trophoblastic disease or ovarian germ cell tumours. We report a case of metastatic cervical squamous cell carcinoma (SCC) presenting with an elevated serum $\beta$-hCG. A 44-year-old Vietnamese lady, para 3, with a past surgical history of a Cesarean section and tubal ligation, was first diagnosed with stage 3B cervical SCC in November 2016 and underwent chemoradiation. She completed her treatment uneventfully and remained disease-free. She then presented to the emergency department in January 2018 complaining of right lower back and abdominal pain for 2 weeks. Her urine pregnancy test was positive and her serum $\beta$-hCG was done which showed an elevated level of $593 \mathrm{IU} / \mathrm{mL}$. Further investigations showed no intra or extra-uterine pregnancy and a magnetic resonance imaging (MRI) of the abdomen and pelvis revealed no pelvic recurrence but presence of a large liver mass and para-aortic lymphadenopathy. Biopsy of the mass confirmed recurrent metastatic cervical SCC. She was not a suitable candidate for surgery and was counseled for palliative chemotherapy. $\beta$-hCG trending revealed a downward trend after commencing on palliative chemotherapy. Ectopic secretion of $\beta$-hCG from tumour cells is a consideration in a patient who has detectable serum $\beta$-hCG but no clinical evidence of pregnancy. Although rare, metastatic or recurrent cervical SCC can secrete serum $\beta$-hCG and case reports have shown presence of serum $\beta$-hCG even in non-gynecological tumours. Larger studies are required to investigate the full potential of serum or urinary $\beta$-hCG in the role of prognosticating SCC of the cervix.
\end{abstract}

Keywords: Cervical cancer; Squamous cell carcinoma; HCG

Manuscript submitted June 3, 2018, accepted June 22, 2018

aDepartment of Obstetrics and Gynecology, KK Women's and Children's Hospital, Singapore 229899, Singapore

bDepartment of Gynecological Oncology, KK Women's and Children's Hospital, Singapore 229899, Singapore

${ }^{\mathrm{c} C o r r e s p o n d i n g ~ A u t h o r: ~ M i c h e l l e ~ E-J y n ~ K w e k, ~} 100$ Bukit Timah Road, KK Women's and Children's Hospital, Singapore 229899, Singapore.

Email: michelle.kwek@mohh.com.sg

doi: https://doi.org/10.14740/jmc3103w

\section{Introduction}

Human chorionic gonadotropin (hCG) is a glycoprotein hormone comprising two subunits, $\alpha$ and $\beta$, and is usually secreted by the trophoblast. An elevated serum beta human chorionic gonadotropin ( $\beta$-hCG) level in a female patient normally demonstrates the presence of a pregnancy or less commonly, gestational trophoblastic disease or ovarian germ cell tumors. Previous in vitro studies performed suggested that $\beta$-hCG promotes tumor progression by inhibiting apoptosis as a result of blocking the transforming growth factor-beta receptor. This has been correlated with the relative rapid progression of $\beta$-hCG positive tumors and greater propensity to metastasize [1]. We report a case of stage $3 \mathrm{~B}$ cervical squamous cell carcinoma (SCC) presenting with abdominal pain and findings of elevated serum $\beta$-hCG with pregnancy excluded. Further investigations revealed a metastasis of her primary cervical SCC. We suggest that this rise in $\beta$-hCG attributed to ectopic secretion of the hormone by tumour cells and could be a potential prognostic and therapeutic marker.

\section{Case Report}

A 44-year-old Vietnamese female, gravida 4, parity 3 (1NVD, 2 LSCS, 1 TOP), with a past surgical history of previous Cesarean section, tubal ligation in 2011, was first diagnosed with stage 3B cervical SCC in November 2016 with right hydronephrosis and a large right sided urinoma. She underwent six cycles of concurrent radiotherapy (external beam radiotherapy $50.4 \mathrm{~Gy} \times 5$ and brachytherapy $\times 3$ (two interstitial and one intracavitary)) and cisplatin. Her treatment duration lasted from December 1, 2016 to January 24, 2017. She remained amenorrheic since completion of treatment. The patient also underwent percutaneous nephrostomy and drainage catheter insertion for her hydronephrosis. Her condition was complicated by thrombosis in right uterine, internal iliac and common iliac veins and she was started on therapeutic clexane. A positron emission tomography-computed tomography (PET-CT) scan and magnetic resonance imaging (MRI) of the pelvis were done in April 2017 to assess treatment response which showed interval metabolic resolution at region of cervix, no cervical 


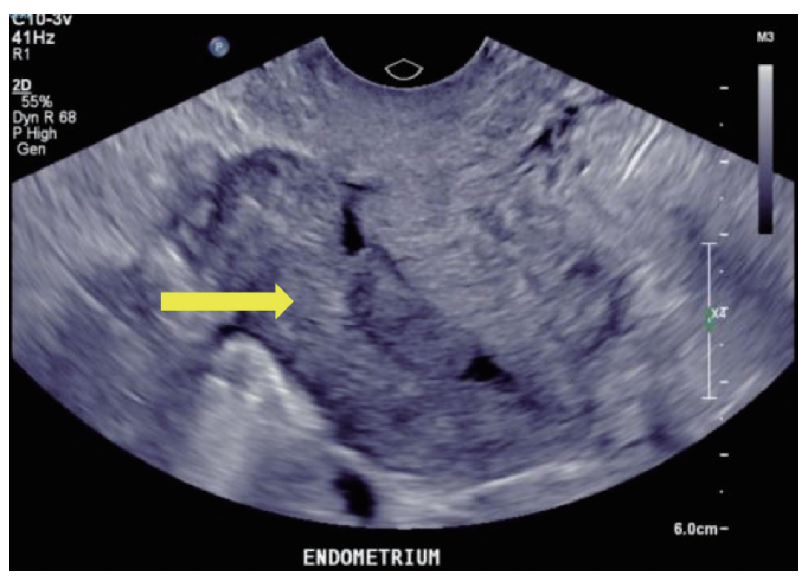

Figure 1. Transvaginal ultrasound scan of the pelvis showing an endometrial polyp (arrow) and absence of intra- and extrauterine pregnancy.

mass seen, resolution of hydronephrosis and no distant metastasis. The previously noted thrombosis in right uterine, internal iliac and common iliac veins had resolved. The patient was then planned for surveillance monitoring. She remained disease-free for 9 months.

In January 2018, she was referred to our emergency department with worsening right lower back and flank pain of 2 weeks duration, and initial urine pregnancy test was positive. Abdominal examination revealed tenderness at the right flank. Cervix was not visualized on speculum examination and no abnormality of the vault was noted. Initial investigations showed an elevated $\beta-\mathrm{hCG}$ of $593 \mathrm{IU} / \mathrm{mL}$.

Ultrasound scan of the pelvis reported a $2.1 \times 0.9 \times 0.9 \mathrm{~cm}$ endometrial polyp and a $2.9 \times 2.8 \times 2.9 \mathrm{~cm}$ left wall intramural fibroid. No gestational sac was noted. Bilateral ovaries were normal, no adnexal mass was seen; there was small amount of free fluid in the pouch of Douglas (Fig. 1). Ultrasound scan of the abdomen revealed a large mass $(13.1 \times 10.8 \times 8.3 \mathrm{~cm})$ occupying the whole right lobe of the liver with two cystic components noted within the mass which appears to be extending beyond the liver capsule. The right hepatic vein was attenuated, suggesting possible diagnosis of hepatic metastasis. We proceeded with a MRI of the abdomen and pelvis which showed large liver lesions with right chest wall involvement and para-aortic lymphadenopathy, suspicious for metastasis (Fig. 2).

The patient subsequently underwent an ultrasound guided needle aspiration and biopsy of the right hepatic lobe cystic lesion. Histology showed features consistent with metastatic squamous cell carcinoma from the female genital tract. The patient was counselled for palliative chemotherapy and was commenced on a cisplatin and taxol regime. Her serum $\beta-\mathrm{hCG}$ revealed a downward trend after completion of palliative chemotherapy.

\section{Discussion}

Human chorionic gonadotropin (hCG) has been detected within tissue homogenates, culture fluid, and serum of patients

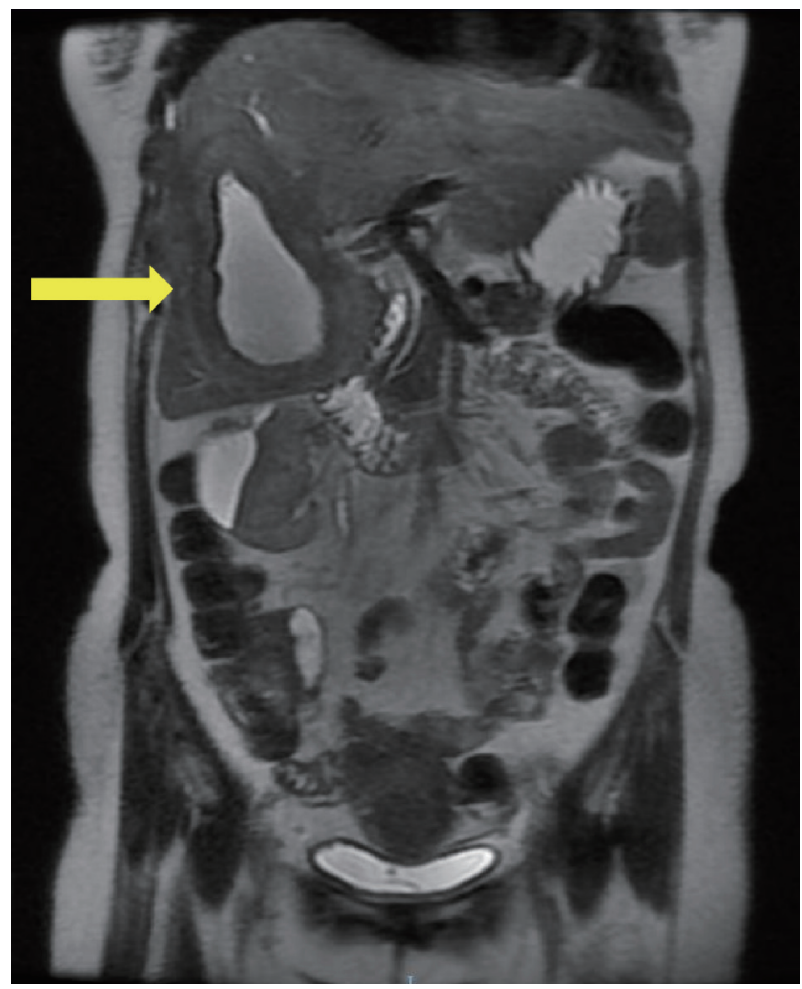

Figure 2. Coronal image MRI of the abdomen and pelvis showing large liver metastases (arrow) and absence of local pelvic recurrence of disease.

with squamous cell carcinoma of the cervix. Studies regarding in vivo localization of beta-hCG in squamous cell carcinoma of the cervix are scant and non-conclusive [2]. In this unique case, the rise in $\beta$-hCG is likely attributed to ectopic secretion by metastatic cervical SCC tumor in the liver, as presence of pregnancy, choriocarcinoma or germ cell tumor were excluded. As early as 1981, it was discovered that cervical cancer can produce a $\beta$-hCG immunoreactive substance [3]. However, only $30 \%$ are detectable in blood as the glycoprotein is usually rapidly degraded and cleared by the liver and kidney [4]. The poor sensitivity has restricted the use of hCG in detecting cervical cancer and monitoring of response following therapy but this has led to the investigation of using urinary gonadotropins (UGF) as an alternative tumor marker. Although there is a lack of correlation between mean level of serum hCG level and advancement of cancer, studies suggest increased sensitivity of serum hCG and UGF with advanced disease $[5,6]$. In vitro studies with bladder cell lines suggest $\beta$-hCG plays an antiapoptotic role and $\beta$-hCG expression correlates with reduced tumor cell apoptosis and may be involved in tumor vascularization and dissemination [7]. This may mean that serum and urinary $\beta$-hCG levels can be useful in screening of patients post therapy and monitoring cancer progression or regression.

\section{Conclusions}

This case has shown a phenomenon of recurrent metastatic 
cervical SCC with elevated serum $\beta$-hCG level originating from the hepatic metastasis. We conclude that ectopic secretion of $\beta$-hCG from tumor cells is a consideration in a patient who has detectable serum $\beta$-hCG but no clinical evidence of pregnancy. Although rare, metastatic or recurrent SCC can secrete serum $\beta$-hCG [8] and case reports have shown presence of serum $\beta$-hCG even in non-gynecological tumors $[9,10]$. Larger studies are required to investigate the full potential of serum or urinary $\beta$-hCG in the role of prognosticating SCC of the cervix.

\section{Grant Support}

None to declare.

\section{Conflict of Interest}

None to declare.

\section{References}

1. Iles RK. Ectopic hCGbeta expression by epithelial cancer: malignant behaviour, metastasis and inhibition of tumor cell apoptosis. Mol Cell Endocrinol. 2007;260262:264-270.

2. Hameed A, Miller DS, Muller CY, Coleman RL, AlboresSaavedra J. Frequent expression of beta-human chorionic gonadotropin (beta-hCG) in squamous cell carcinoma of the cervix. Int J Gynecol Pathol. 1999;18(4):381-386.

3. Cole LA, Hussa RO, Rao CV. Discordant synthesis and secretion of human chorionic gonadotropin and subunits by cervical carcinoma cells. Cancer Res. 1981;41(5):16151619.

4. Cole LA, Kardana A, Andrade-Gordon P, Gawinowicz MA, Morris JC, Bergert ER, O'Connor J, et al. The heterogeneity of human chorionic gonadotropin (hCG). III. The occurrence and biological and immunological activities of nicked hCG. Endocrinology. 1991;129(3):15591567.

5. Cole LA, Wang YX, Elliott M, Latif M, Chambers JT, Chambers SK, Schwartz PE. Urinary human chorionic gonadotropin free beta-subunit and beta-core fragment: a new marker of gynecological cancers. Cancer Res. 1988;48(5):1356-1360.

6. Crawford RA, Iles RK, Carter PG, Caldwell CJ, Shepherd $\mathrm{JH}$, Chard T. The prognostic significance of beta human chorionic gonadotrophin and its metabolites in women with cervical carcinoma. J Clin Pathol. 1998;51(9):685688.

7. Li D, Wen X, Ghali L, Al-Shalabi FM, Docherty SM, Purkis P, Iles RK. hCG beta expression by cervical squamous carcinoma - in vivo histological association with tumour invasion and apoptosis. Histopathology. 2008;53(2):147-155.

8. Mustafa A, Bozdag Z, Tepe NB, Ozcan HC. An unexpected reason for elevated human chorionic gonadotropin in a young woman. Cervical squamous carcinoma. Saudi Med J. 2016;37(8):905-907.

9. Demirtas E, Krishnamurthy S, Tulandi T. Elevated serum beta-human chorionic gonadotropin in nonpregnant conditions. Obstet Gynecol Surv. 2007;62(10):675-679; quiz 691.

10. Turner JH, Ross H, Richmon J. Secretion of beta-HCG from squamous cell carcinomas of the head and neck. Otolaryngol Head Neck Surg. 2010;143(1):169-170. 